\title{
Throughput-Reliability Tradeoff in Decode-and-Forward Cooperative Relay Channels: A Network Information Theory Approach
}

\author{
Jun Li, and Wen Chen \\ Department of Electronic Engineering, \\ Shanghai Jiao Tong University, \\ Shanghai, China. 200240. \\ Email: $\{$ jleesr80,wenchen $\} @$ sjtu.edu.cn
}

\begin{abstract}
Cooperative transmission protocols are always designed to reach the largest diversity gain and the largest network capacity simultaneously. The concept of diversity-multiplexing tradeoff (DMT) in MIMO systems put forward by Zheng and Tse has been extended to this field. However, the concept of multiplexing gain in DMT constrains a better understanding of the asymptotic interplay between transmission rate, frame error probability (FEP) and signal-to-noise ratio (SNR), and also fails to predict FEP curves accurately. Two improved methods are then put forward. One is by Narasimhan who proposes finite-SNR diversity-multiplexing gain tradeoff which gives a tighter lower bound of the FEP curves by applying nonlinear programming in MIMO systems, and the other is by Azarian and Gamal who propose a new rule called the throughput-reliability tradeoff (TRT) which avoids the limitation of the conception of multiplexing and elucidates the linearly asymptotic trends exhibited by the FEP curves in block-fading MIMO channels. The finite-SNR diversity-multiplexing gain tradeoff has already been applied to cooperative relay channels. However, this method is time-consuming in computation since nonlinear programming is used, especially in large networks. In this paper, we will use TRT rule to give the relationship between transmission rate, FEP and SNR in decode-and-forward (DF) cooperative protocols. We also exhibit the FEP curves predicted by TRT. To do this, We first propose a symbol based slotted decode-and-forward (SSDF) protocol as the infrastructure. Network information theory is also used to bound the capacity of the protocol.
\end{abstract}

\section{INTRODUCTION}

Recently, there has been a growing interest in the design and analysis of protocols in cooperative transmission systems [1]-[5], [15], [17]-[20]. Such a system can be viewed as a derivative form of MIMO system. As well known, MIMO is generally used for increasing the amount of diversity to combat channel fading or the number of the degrees of freedom [6][9]. Under some particular arrangement, e.g., with clustered and full-duplex relays, cooperative channels can mimic the MIMO channels gracefully [20]. On the other hand, network information theory has been studied for almost 30 years, which focus on the achievable rates and capacity region in various network channels [10]-[15], such as relay channels, broadcast channels and so on. Thus, from a new perspective of combining MIMO with network information theory, the designer of protocols in cooperative transmission systems should pay attention to not only the diversity gain but also the network capacity.

In [16], Zheng and Tse give an formulation between the diversity gain and multiplexing gain by using the Gaussian code. The same derivation method can be extended to any general code, which is called diversity-multiplexing tradeoff (DMT). The DMT assumes a family of codes, in which diversity gain $d$ and multiplexing gain $r$ are defined by

$$
d \triangleq-\lim _{\rho \rightarrow \infty} \frac{\log \left(P_{e}(\rho)\right)}{\log \rho} \quad \text { and } \quad r \triangleq \lim _{\rho \rightarrow \infty} \frac{R(\rho)}{\log \rho},
$$

where $\rho, P_{e}(\rho)$ and $R(\rho)$ represent the signal-to-noise ratio (SNR), frame error probability (FEP) and transmission rate respectively. So a scheme's DMT means that at the $r$ multiplexing gain, the diversity gain that the scheme acquires should not exceed $d(r)$. There must be a tradeoff between diversity gain and multiplexing gain in MIMO systems among various coding schemes. Now, this elegant formulation is successfully used as a standard in cooperative communication systems to evaluate the performance of different cooperative transmission protocols [17]-[20].

Due to the limitation posed by DMT, Another two improved methods have been put forward. One is by Narasimhan [21] who focuses on the finite-SNR diversity-multiplexing tradeoffs. He proposes the outage probability curves in correlated Rayleigh and Rician MIMO channels by using nonlinear programming on the condition that SNR is finite. New definition of diversity gain and multiplexing gain are also given in [21],

$$
d \triangleq-\frac{\partial \ln P_{o}(\rho)}{\partial \ln \rho} \quad \text { and } \quad r \triangleq \frac{R}{\log _{2}(1+\rho)},
$$

where $P_{o}$ is the outage probability. Thus, the diversity gain defined at finite SNR is the slope of the $\log P_{o}$ vs $\log \rho$ curves. This method has been extended to cooperative relay channels [22] where one source, one relay and one destination are taken into account. Since the nonlinear programming is used to work out the $P_{o}$, the process of computation is time-consuming, especially in large networks.

The other improvement is proposed by Azarian and Gamal [23]. They pointed out the limitation of DMT imposed by 
the concept of multiplexing gain, that is, it will lead to a malfunction in predicting the FEP curves due to

$$
\limsup _{\rho \rightarrow \infty} \frac{R}{\log \rho} \neq \liminf _{\rho \rightarrow \infty} \frac{R}{\log \rho} .
$$

Meanwhile, They put forward a relationship between the three quantities $\left\{R, \log \rho, P_{e}(R, \rho)\right\}$, which is called throughputreliability tradeoff (TRT) [23] where the reliability gain has the the same meaning with the finite-SNR diversity gain defined in [21]. From the simulations in [23], we can see that TRT predicts the FEP curves by using the linear approximation method, which implies that it is less computational than the first method.

For the sake of the computational simplicity, especially in lager networks, we pay attention to the TRT formulation. In this paper, we consider the decode-and-forward (DF) cooperative protocol with arbitrary number of nodes. In the DF protocol, relays receive the signals from source according to some timing sequence arranged beforehand and then decode them before retransmit them to destination, which is different from amplify-and-forward (AF) protocol where relays retransmit signals received from source without decoding them. An important factor to distinguish various protocols is the scheduling strategy used to arrange the receiving and transmitting orders of relays. Among various strategies, a simple and efficient strategy is the one that used in slotted amplify-and-forward (SAF) protocol [19]. Another well-known AF protocol is non-orthogonal amplify-and-forward (NAF) protocol [3], [17], which can be seen as a special case of SAF. Obviously, these scheduling strategies in AF protocols can also be transplanted to DF protocols.

To give the TRT relationship in DF protocols, we should:

1) find out the difference between DF cooperative channels and MIMO channels: The difference between DF cooperative channels and MIMO channels is that there are hops (relays) between the source and destination in the former while the source and destination are connected directly in the latter. How to deal with these hops in channels' model is important. Network information theory is used to bound the protocols' capacity [14]. Outage region is thus defined. By the conclusion in [14], the capacity bound of networks with DF protocols and arbitrary topologies can be work out. While in this paper, we only focus on one hop between source and destination.

2) set up a simple and common DF protocol as infrastructure to ease the analysis: We apply the round-rubin scheduling strategy used in SAF to the DF protocol so that we establish the symbol based slotted DF (SSDF) protocol which is detailedly introduce in the sequel. Even we analyze the TRT formulation based on SSDF protocol, the same analytical method can be extended to any other DF protocols.

3) exhibit the TRT relationship in SSDF protocol: We extend the TRT analytical method proposed in [23] here after outage regions are determinated in SSDF by using capacity bounding technology given in [14]. Simulations are also presented. From numerical results, we can see the asymptotic trends predicted by TRT which linearly approximates to the simulating curves.

We emphasize that, in quasi-static fading channels, codes that approach capacity are typically used on each packet such that the FEP is well approximate by the channels outage probability [21]. So in this paper, we only focus on the outage probability.

The rest of the paper is organized as follows. In section ??, we make a general view on the scheduling strategy's description of SAF protocol, and then we propose a symbol based SDF protocol. TRT analysis on SSDF is deployed in section III, where we prove the TRT formulation. Section IV demonstrates the rule though numerical results, which shows that besides MIMO systems, the concept of TRT is also holding in cooperative AF protocols.

The notations used in this paper go as follows. $(x)^{+}$denotes $\max \{0, x\},(x)^{-}$denotes $\min \{0, x\}, \mathbb{R}^{N}$ and $\mathbb{C}^{N}$ means the set of real and complex $N$-tuples, and $\mathbb{R}^{N+}$ denotes the set of non-negative $N$-tuples. If some set $\mathcal{O} \subseteq \mathbb{R}^{N}$, we denote the complete set of $\mathcal{O}$ as $\mathcal{O}^{c}$, while $\mathcal{O} \cap \mathbb{R}^{N+}$ as $\mathcal{O}^{+} . \boldsymbol{\Lambda}_{\text {x }}$ denotes the auto-covariance matrix of vector $\boldsymbol{x}$.

\section{SySTEM MODEL}

For the sake of understanding, we give a simple model description of SAF protocols. For more details, refer to [19]. Note that in Fig. 1, dashed boxes mean the receiving procedure while the solid ones mean transmitting.

In the SAF protocol (see Fig. 1), the superframe is composed of $(N+1)$ cooperative frames, and each cooperative frame is transmitted in a time slot by the source.

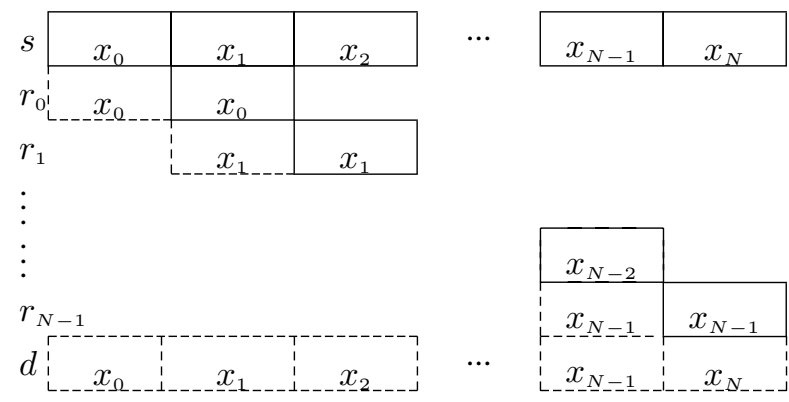

Fig. 1. Frame structure and scheduling strategy in SAF protocol.

Consider the extreme case where each cooperative frame in SAF protocol only contains a single symbol, and the isolated relays are arranged by round-robin scheduling strategy [19]. Each relay decodes the received signals and retransmits them. Then we get the SSDF protocol naturally. By using relays circularly, SSDF can deal with $l>N+1$, where $l$ is the frame length.

Without loss of generality, we establish a SSDF cooperative channel model with one source, one destination and $N$ relays. Each node is constrained by half-duplexing and each relay is isolated from the other relays. All channels are assumed to be flat Rayleigh-fading and quasi-static in at least one frame period, all nodes are in half-duplex mode, and all noises 


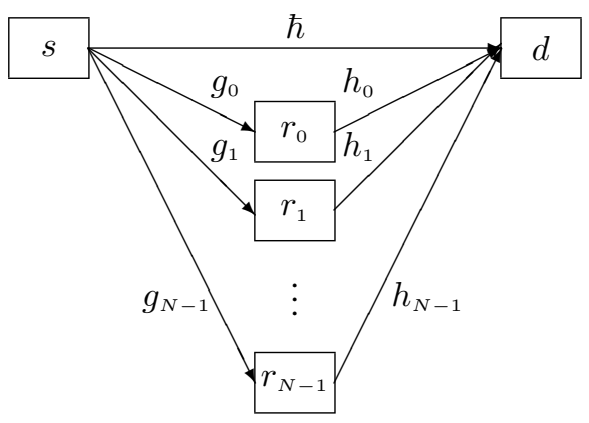

Fig. 2. Cooperative channels with $N$ isolated relays.

observed by relays and destination are Gaussian distribution.

Furthermore, we use $g_{i}, \hbar, h_{i}$ and $k_{i, j}$ to denote the channels between the source and the $i$-th relay, the source and the destination, the $i$-th relay and the destination, the $i$-th relay and the $j$-th relay respectively (see Fig. 2), which are all Rayleigh distribution with zero mean and variance $\sigma_{g_{i}}^{2}, \sigma_{\hbar}^{2}, \sigma_{h_{i}}^{2}, \sigma_{k_{i j}}^{2}$ respectively. We take $\sigma_{g_{i}}^{2}=\sigma_{\hbar}^{2}=\sigma_{h_{i}}^{2}=\sigma_{k_{i j}}^{2}=1$ in the numerical examples. All noises observed by the relay and destination have zero mean and variance $\sigma_{r_{j}}^{2}(j=0,1,2, \cdots, N-1)$ and $\sigma_{d}^{2}$. We assume that $\sigma_{r_{j}}^{2}=\sigma_{d}^{2}=\sigma^{2}$. When relays are isolated from each other, the channels coefficients $k_{i, j}=0$. Similar to the power allocation in [22], we denote $P$ as the average total network transmit power over a frame, that is,

$$
\begin{aligned}
& \mathcal{E}\left\{\frac{1}{l} \sum_{i=0}^{l-1}\left|x_{s, i}\right|^{2}\right\}=\kappa P \\
& \mathcal{E}\left\{\frac{1}{l} \sum_{i=0}^{l-1}\left|x_{r_{j}, i}\right|^{2}\right\}=\tau_{j} P(j=0,1,2, \cdots, N-1) \\
& \kappa+\sum_{j=0}^{N-1} \tau_{j}=1 \quad \text { and } \quad \kappa, \tau_{0}, \cdots, \tau_{N-1} \geq 0,
\end{aligned}
$$

where $x_{s, i}$ denotes the $i$-th symbol in a frame transmitted by source, and $x_{r_{j}, i}$ denotes the $i$-th symbol in a frame transmitted by the $j$-th relay. Based on the power constraint in (4), we define the network SNR as

$$
\rho \triangleq \frac{P}{\sigma^{2}} .
$$

By applying these definitions, we then give some preliminary theoretical results that will be used in the sequel.

The bounds in Lemma ?? can facilitate the definition of outage regions in SSDF protocol, which will be analyzed in details in the following section by applying network information theory.

\section{OUtAge Regions in SSDF Protocol: A Network INFORMATION THEORY APPROACH}

An achievable capacity region of large networks has been worked out by network information theory [14] where multihop are taken into account with decoded strategies used in each relay. [14] concludes the common results of capacity regions in arbitrary topologized networks (feedback is not considered). Results in [10], [13] can be seen as a special cases of [14]. By the conclusions in [14], we get the maximum capacity of SSDF protocol,

$$
I_{\max } \leq \max \left\{\min \left\{I\left(X_{s} ; Y_{r}\right), I\left(X_{s}, X_{r} ; Y_{d}\right)\right\}, I\left(X_{s} ; Y_{d}\right)\right\},
$$

where $X$ denotes the transmitted signals in each node, and $Y$ denotes the received signals in each node. Since there are $N$ relays in SSDF protocol. So $X_{r}=\left(X_{r, 0}, X_{r, 1}, \cdots, X_{r, N-1}\right)$ and $Y_{r}=\left(Y_{r, 0}, Y_{r, 1}, \cdots, Y_{r, N-1}\right)$.

In non-ergodic fading channels, performance of the connection is evaluated in terms of outage probability, which is defined as the event that the instantaneous mutual information does not support the intended rate [25], [26], i.e.,

$$
\mathcal{O}_{p} \triangleq\{\boldsymbol{H} \mid I(x ; y \mid \mathbf{H}=\boldsymbol{H})<R\},
$$

where $\boldsymbol{H}$ is a channel realization. The lower bound of $\mathcal{O}_{p}$ 's probability is defined as outage probability $P_{o}(R, \rho)$ [25], [26]. Therefore,

$$
\begin{aligned}
P_{o}(R, \rho) & =\inf _{\boldsymbol{\Lambda}_{\mathbf{x}}} \operatorname{Pr}\left\{\mathcal{O}_{p}\right\} \\
& =\operatorname{Pr}\left\{\max _{\boldsymbol{\Lambda}_{\mathbf{x}}} I(x ; y \mid \boldsymbol{H})<R\right\} .
\end{aligned}
$$

So under some channel realization $\boldsymbol{H}$, there are three outage events according to (6),

$$
\begin{aligned}
& E_{s ; r} \quad: I\left(X_{s} ; Y_{r} \mid \boldsymbol{H}\right)=\sum_{i=0}^{l-2} \log \left(1+\kappa \rho\left|g_{i_{N}}\right|^{2}\right)<l R \\
& E_{s, r ; d}: I\left(X_{s}, X_{r} ; Y_{d} \mid \boldsymbol{H}\right)=\log \left(1+\kappa \rho|\hbar|^{2}\right)+ \\
& \sum_{i=0}^{l-2} \log \left(1+\kappa \rho|\hbar|^{2}+\frac{N l \tau_{i_{N}}}{l-1} \rho\left|h_{i_{N}}\right|^{2}\right)<l R \\
& E_{s ; d} \quad: I\left(X_{s} ; Y_{d} \mid \boldsymbol{H}\right)=\log \left(1+\kappa \rho|\hbar|^{2}\right)<R .
\end{aligned}
$$

Then from (6), we conclude that the outage event of the whole network with SSDF protocol is

$$
\begin{array}{r}
E_{o}: \max \left\{\min \left\{I\left(X_{s} ; Y_{r} \mid \boldsymbol{H}\right), I\left(X_{s}, X_{r} ; Y_{d} \mid \boldsymbol{H}\right)\right\},\right. \\
\left.I\left(X_{s} ; Y_{d} \mid \boldsymbol{H}\right)\right\}<l R,
\end{array}
$$

that is,

$$
E_{o}=\left(E_{s ; r} \cup E_{s, r ; d}\right) \cap E_{s ; d} .
$$

From (9), we notice that $E_{s ; r}$ is irrelevant with $E_{s, r ; d}$ and $E_{s ; d}$, and $E_{s, r ; d} \subseteq E_{s ; d}$. So

$$
\begin{aligned}
P\left(E_{o}\right) & =P\left(\left(E_{s ; r} \cup E_{s, r ; d}\right) \cap E_{s ; d}\right) \\
& =P\left(\left(E_{s ; r} \cap E_{s ; d}\right) \cup E_{s, r ; d}\right) \\
& =P\left(E_{s ; r}\right) P\left(E_{s ; d}\right)+P\left(E_{s, r ; d}\right)-P\left(E_{s ; r}\right) P\left(E_{s, r ; d}\right) \\
& =P\left(E_{s ; r}\right)\left(P\left(E_{s ; d}\right)-P\left(E_{s, r ; d}\right)\right)+P\left(E_{s, r ; d}\right) .
\end{aligned}
$$

(12) shows that $P\left(E_{o}\right)$ is a increasing function of $P\left(E_{s ; r}\right)$, $P\left(E_{s, r ; d}\right)$ and $P\left(E_{s ; d}\right)$. According to Lemma ??, when $\rho \rightarrow$ 
$\infty$, the lower bounds of the three probability values are

$$
\begin{gathered}
P\left(E_{s ; r}\right) \geq \operatorname{Pr}\left\{\sum_{i=0}^{l-2} \log \left(1+\rho\left|g_{i_{N}}\right|^{2}\right)<l R\right\}, \\
P\left(E_{s, r ; d}\right) \geq \operatorname{Pr}\left\{\operatorname { m a x } \left\{\log \left(1+\rho|\hbar|^{2}\right)^{l},\right.\right. \\
\left.\left.\log \left(1+\rho|\hbar|^{2}\right)+\sum_{i=0}^{l-2}\left(1+\rho\left|h_{i_{N}}\right|^{2}\right)\right\} \stackrel{\circ}{<} l R\right\}, \\
P\left(E_{s ; d}\right) \geq \operatorname{Pr}\left\{\log \left(1+\rho|\hbar|^{2}\right)<R\right\},
\end{gathered}
$$

and the upper bounds are

$$
\begin{aligned}
& P\left(E_{s ; r}\right) \leq \operatorname{Pr}\left\{\sum_{i=0}^{l-2} \log \left(1+\frac{l \rho}{2 l-1}\left|g_{i_{N}}\right|^{2}\right)<l R\right\}, \\
& P\left(E_{s, r ; d}\right) \leq \operatorname{Pr}\left\{\operatorname { m a x } \left\{\log \left(1+\frac{l \rho}{2 l-1}|\hbar|^{2}\right)^{l},\right.\right. \\
& \left.\left.\log \left(1+\frac{l \rho}{2 l-1}|\hbar|^{2}\right)+\sum_{i=0}^{l-2}\left(1+\frac{l \rho}{2 l-1}\left|h_{i_{N}}\right|^{2}\right)\right\}<l R\right\}, \\
& P\left(E_{s ; d}\right) \leq \operatorname{Pr}\left\{\log \left(1+\frac{l \rho}{2 l-1}|\hbar|^{2}\right)<R\right\} .
\end{aligned}
$$

We define another outage event $E_{r ; d}$, which satisfy that $E_{s, r ; d}=E_{s ; d} \cap E_{r ; d}$. From (13) and (14), we get the lower bound of $P\left(E_{r ; d}\right)$, that is,

$P\left(E_{r ; d}\right) \geq \operatorname{Pr}\left\{\log \left(1+\rho|\hbar|^{2}\right)+\sum_{i=0}^{l-2}\left(1+\rho\left|h_{i_{N}}\right|^{2}\right)<l R\right\}$,

and the upper bound

$P\left(E_{r ; d}\right) \leq$

$\operatorname{Pr}\left\{\log \left(1+\frac{l \rho}{2 l-1}|\hbar|^{2}\right)+\sum_{i=0}^{l-2}\left(1+\frac{l \rho}{2 l-1}\left|h_{i_{N}}\right|^{2}\right)<l R\right\}$

So we rewrite the network outage probability of (12) as

$$
P_{o}=P\left(E_{o}\right)=P\left(\left(E_{s ; r} \cup E_{r ; d}\right) \cap E_{s ; d}\right) .
$$

Based on these conclusions, we then make a more detailed TRT analysis on the SSDF protocols.

\section{Throughrut-REliability TRADEOFF ANALYSIS OF SSDF PROTOCOL}

The asymptotic relationship of $R, \rho$ and $P_{o}(R, \rho)$ has been well derived in MIMO channels [23], [24]. Our work proves that such relationship also holds in SSDF protocol.

Theorem 1: For the one source, one destination and $N$ relays SSDF block-fading cooperative channels with $l(l \geq$ $N+1)$ symbols per frame, there are $k(N \geq k \geq 0, k \in \mathbb{Z})$ operating regions, in which

$$
\lim _{\substack{\rho \rightarrow \infty \\(R, \rho) \in \mathcal{R}(k)}} \frac{\log P_{o}(R, \rho)-c(k) R}{\log \rho}=-g(k),
$$

where $\mathcal{R}(k)$ means the $k$-th operating region, $g(k)$ is referred to the reliability gain coefficient and $\frac{g(k)}{c(k)}$ is referred to the throughput gain coefficient.

Consider two cases, $(l-1)_{N}=0$ and $(l-1)_{N} \neq 0$.

4) $(l-1)_{N}=0$ : The $k$ regions can be combined to two super operating regions, i.e.,

$$
\begin{aligned}
& \mathcal{R}(k) \\
& \triangleq \begin{cases}\left\{(R, \rho) \mid \frac{(l-1)(k+1)}{l N}>\frac{R}{\log \rho}>\frac{(l-1) k}{l N}\right\}, & N>k \geq 0, \\
\left\{(R, \rho) \mid 1>\frac{R}{\log \rho}>\frac{l-1}{l}\right\}, & k=N,\end{cases}
\end{aligned}
$$

and $\{c(k), g(k)\}$ are defined according to $\mathcal{R}(k)$,

$$
\{c(k), g(k)\} \triangleq \begin{cases}\left\{1+\frac{l N}{l-1}, 1+N\right\}, & N>k \geq 0 \\ \{1,1\}, & k=N\end{cases}
$$

5) $(l-1)_{N}=m(0<m<N)$ : There are two extreme cases, i.e., the remaining $m$ symbols are transferred through the best $m$ relay channels and the worst $m$ relay channels. They give the two bounds of the outage probability curves. When the best $m$ source-relay channels are first used (BCFU), there are three super regions:

$$
\begin{aligned}
& \mathcal{R}_{1}(k) \\
& \triangleq\left\{\begin{array}{cl}
\left\{(R, \rho) \mid \frac{(l-1+N-m)(k+1)}{l N}>\right. & m>k \geq 0, \\
\left.\frac{R}{\log \rho}>\frac{(l-1+N-m) k}{l N}\right\}, & \\
(R, \rho) \mid \frac{(l-1-m)(k+1)+m N}{l N}> & \\
\left.\frac{R}{\log \rho}>\frac{(l-1-m) k+m N}{l N}\right\}, & N>k \geq m, \\
\left\{(R, \rho) \mid 1>\frac{R}{\log \rho}>\frac{l-1}{l}\right\}, & k=N,
\end{array}\right.
\end{aligned}
$$

and $\left\{c_{1}(k), g_{1}(k)\right\}$ are defined according to $\mathcal{R}_{1}(k)$, that is,

$$
\begin{aligned}
& \left\{c_{1}(k), g_{1}(k)\right\} \\
& \triangleq \begin{cases}\left\{1+\frac{l N}{l-1+N-m}, 1+N\right\}, & m>k \geq 0, \\
\left\{1+\frac{l N}{l-1-m}, 1+\frac{(l-1) N}{l-1-m}\right\}, & N>k \geq m, \\
\{1,1\}, & k=N .\end{cases}
\end{aligned}
$$

When the worst $m$ source-relay channels are first used (WCFU), then

$$
\begin{aligned}
& \mathcal{R}_{2}(k) \\
& \triangleq\left\{\begin{array}{cl}
\left\{(R, \rho) \mid \frac{(l-1-m)(k+1)}{l N}>\right. & N-m>k \geq 0, \\
\left.\frac{R}{\log \rho}>\frac{(l-1-m) k}{l N}\right\}, & \\
(R, \rho) \mid \frac{(l-1+N-m)(k+1)+m N-N^{2}}{l N} & N>k \geq N-m, \\
>\frac{R}{\log \rho}> & \left.\frac{(l-1+N-m) k+m N-N^{2}}{l N}\right\}, \\
\left\{(R, \rho) \mid 1>\frac{R}{\log \rho}>\frac{l-1}{l}\right\}, & k=N,
\end{array}\right.
\end{aligned}
$$


and $\left\{c_{2}(k), g_{2}(k)\right\}$ are defined according to $\mathcal{R}_{2}(k)$, that is,

$$
\begin{aligned}
& \left\{c_{2}(k), g_{2}(k)\right\} \\
& \triangleq \begin{cases}\left\{1+\frac{l N}{l-1-m}, 1+N\right\}, & N-m>k \geq 0, \\
\left\{1+\frac{l N}{l-1+N-m}, 1+\frac{(l-1) N}{l-1+N-m}\right\}, & N>k \geq N-m, \\
\{1,1\}, & k=N .\end{cases}
\end{aligned}
$$

\section{CONCLUSiON}

We base our work on the Azarian and Gamal's elegant formulation concluded from MIMO systems to throw a light on the asymptotic interplay between $R, \log \rho$ and $P_{o}(R, \rho)$ in DF protocols. To do this, we first find out the differences between DF cooperative channels and MIMO channels, i.e., in DF cooperative channels there are at least one hop between source and destination while in MIMO channels the source and destination are directly connected. There are many scheduling strategies in cooperative protocols which have nothing to do with the modes relays use to deal with the received signals from source. So these scheduling strategies can be applied to both AF and DF protocols. The scheduling strategy in SAF is an efficient one which can be planted to DF. By applying this scheduling strategy, we get SSDF protocol which is used as our infrastructure to study TRT rule in DF protocols. Then we use network information theory to get the SSDF's network capacity. According to the network's topology of SSDF protocol, three outage events are defined and outage regions can thus be acquired. The outage regions are imperative to work out the outage probability $P_{o}(R, \rho)$. At last we deduce the TRT formulation on the established network outage regions of SSDF protocol to reveal the relationship between $R, \log \rho$, $P_{o}(R, \rho)$. We predict that the same deduction method can be used in other one hop and multi-hop DF protocols with different DMT since network information theory gives the capacity regions of arbitrary topologized networks by which networks' outage regions can thus be worked out.

\section{ACKNOWLEDGMENT}

This work is supported by NSF China \#60672067, by NSF Shanghai \#06ZR14041, by Shanghai-Canada NRC \#06SN07112, by Cultivation Fund of the Key Scientific and Technical Innovation Project, Ministry of Education of China \#706022, by Program for New Century Excellent Talents in University \#NCET-06-0386, and by PUJIANG Talents \#07PJ4046.

\section{REFERENCES}

[1] J. N. Laneman and G. W. Wornell, "Distributed space-time-coded protocols for exploiting cooperative diversity in wireless networks," IEEE Trans. Inf. Theory, vol. 49, no. 10, pp. 2415-2425, Oct. 2003.

[2] J. N. Laneman, D. N. C. Tse, and G. W. Wornell, "Cooperative diversity in wireless networks: Efficient protocols and outage behavior," IEEE Trans. Inf. Theory, vol. 51, no. 12, pp. 3062-3080, Dec. 2004.

[3] R. U. Nabar, F. W. Kneubuhler, and H. Bölcskei, "Performance limits of amplify-and-forward based fading relay channels," Proc. IEEE Int. Conf. Acoustics, Speech and Signal Processing, vol. 4, Montreal, QC, Canada, May 2004, pp. 565-568.
[4] R. U. Nabar, H. Bölcskei, and F. W. Kneubuhler, "Fading relay channels: Performance limits and space-time signal design," IEEE J. Sel. Areas Commun., vol. 22, no. 6, pp. 1099-1109, Aug. 2004.

[5] M. Janani, A. Hedayat, T. Hunter, and A. Nosratinia, "Coded cooperation in wireless communications: Space-tmie transmission and iterative decoding," IEEE Trans. Signal Process., vol. 52, no. 2, pp. 362-371, Feb. 2004

[6] S. Alamouti, "A simple transmitter diversity scheme for wireless communications," IEEE J. Sel. Area Commun., vol. 16, no. 8, pp. 1451-1458, Oct. 1998.

[7] G. Foschini, G. Golden, R. Valenzuela, and P. Wolniansky, "Simplified processing for high spectral efficiency wireless communication employing multi-element arrays," IEEE J. Sel. Area Commun., vol. 17, no. 11, pp. 1841-1852, Nov. 1999.

[8] V. Tarokh, H. Jafarkhani, and A. R. Calderbank, "Space-time codes for high data rate wireless communication: Performance criterion and code construction," IEEE Trans. Inf. Theorey, vol. 44, no. 2, pp. 744-765, Mar. 1998.

[9] V. Tarokh, H. Jafarkhani, and A. R. Calderbank, "Space-time block code from orthogonal designs," IEEE Trans. Inf. Theorey, vol. 45, no. 4, pp. 1456-1467, Sep. 1999.

[10] T. M. Cover and A. A. El-Gamal, "Capacity theorems for the relay channel," IEEE Trans. Inf. Theorey, vol. 25, no. 5, pp. 572-584, Sep. 1979.

[11] T. M. Cover and G. S. L. Leung, "An achievable rate region for the multiple-access channel with feedback," IEEE Trans. Inf. Theorey, vol. 27, no. 3, pp. 292-298, May. 1981.

[12] L. H. Ozarow, "The capacity of the white gaussian multiple access channel with feedback," IEEE Trans. Inf. Theorey, vol. 30, no. 4, pp. 623-629, Jul. 1984.

[13] T. M. Cover, "Comments on broadcast channels," IEEE Trans. Inf. Theorey, vol. 44, no. 6, pp. 2524-2530, Oct. 1998.

[14] P. Gupta, P. R. Kumar, "Towards an Information Theory of Large Networks: An Achievable Rate Region," IEEE Trans. Inf. Theorey, vol. 49, no. 8, pp. 1877-1893, Aug. 2003.

[15] G. Kramer, M. Gastpar, and P. Gupta, "Cooperative strategies and capacity theorems for relay networks," IEEE Trans. Inf. Theorey, vol. 51, no. 9, pp. 3037-3063, Sep. 2005

[16] L. Zheng and D. N. C. Tse, "Diversity and multiplexing: A fundamental tradeoff in multiple antenna channels," IEEE Trans. Inf. Theorey, vol. 49, no. 5, pp. 1073-1096, May 2003.

[17] K. Azarian, H. El-Gamal, and P. Schniter, "On the achievable diversitymultiplexing tradeoff in half-duplexing cooperative channels," IEEE Trans. Inf. Theorey, vol. 51, no. 12, pp. 4152-4172, Dec. 2005.

[18] S. Yang and J.-C. Belfiore, "Optimal space-time codes for the MIMO amplify-and-forward cooperative channel," IEEE Trans. Inf. Theorey, vol. 53, no. 2, pp. 647-663, Feb. 2007.

[19] S. Yang and J.-C. Belfiore, "Towards the optimal amplify-and-forward cooperative diversity scheme," submitted to IEEE Trans. Inf. Theorey, Mar. 2006.

[20] M. Yuksel and E. Erkip, "Cooperative wireless systems: A diversitymultiplexing tradeoff perspective," submitted to IEEE Trans. Inf. Theorey, Sep. 2006.

[21] R. Narasimhan, "Finite-SNR Diversity-Multiplexing Tradeoff for Correlated Rayleigh and Rician MIMO Channels," IEEE Trans. Inf. Theorey, vol. 52, no. 9, pp. 3965-3979, Sep. 2006.

[22] E. Stauffer, Ö. Oyman, R. Narasimhan, and A. Paulraj, "Finite-SNR Diversity-Multiplexing Tradeoffs in Fading Relay Channels," IEEE J. Sel. Areas Commun., vol. 25, no. 2, pp. 245-257, Feb. 2007.

[23] K. Azarian, H. El-Gamal, "The throughput-reliability tradeoff in blockfading MIMO channels," IEEE Trans. Inf. Theorey, vol. 53, no. 2, pp. 488-501, Feb. 2007

[24] K. Azarian, "Outage limited cooperative channels: Protocols and analysis," Ph.D. dissertation, Ohio State Univ., Columbus, OH, 2006.

[25] L. Ozarow, S. Shamai, and A. Wyner, "Information-theoretic considerations in cellular mobile radio," IEEE Trans. Veh. Technol., vol. 43, no. 3, pp. 359-378, May 1994.

[26] İ. E. Telatar, "Capacity of multi-antenna Gaussian channels," Europ. Trans. Telecommun., vol. 10, pp. 585-595, Nov./Dec. 1999.

[27] X. Zhang, Matrix Analysis and Applications, Bei Jing: Tsinghua Univ., 2004.

[28] R. A. Horn and C. R. Johnson, Matrix Analysis, New York: Cambridge, 1985. 